\title{
News and Views December 2020
}

Published online: 29 October 2020

(C) The Regenerative Engineering Society 2020

\section{Awards and Recognitions}

\section{Nobel Prize in Physiology or Medicine}

The 2020 Nobel Prize in Physiology or Medicine was awarded to Charles M. Rice, Harvey J. Alter, and Michael Houghton as announced by The Nobel Assembly at Karolinska Institute, Stockholm. This joint award was granted for the discovery of the Hepatitis $\mathrm{C}$ virus. Later, the discovery led to the sensitive detection of hepatitis through a blood test that is now well accessible around the world and enabled the eradication of post-transfusion hepatitis.

Dr. Charles M. Rice is the Maurice R. and Corinne P. Greenberg Professor in Virology and Head of the Laboratory of Virology and Infectious Disease at the Rockefeller University. His lab has been a leading research group in the area of viral diseases for the past three decades. He was the first who cultured viruses and characterized the viral genome in the mid-1990s. A while later, he successfully produced infectious viruses in the laboratory scale. He made it possible to develop assays for testing drugs with the potential to stop viral replication. This technology was enabled by his work on the development of sub-genomic amplicons of the virus that was capable of replication within the cells. His developed drug was the first of its kind that received FDA approval by 2013 and is known as one of the most effective drugs in treating hepatitis $\mathrm{C}$.

Dr. Harvey J. Alter is a Senior Scholar at the National Institute of Health Clinical Center's Department of Transfusion Medicine. His research was focused on hepatitis in patients who received a blood transfusion. When hepatitis B was discovered in the 1970s, Dr. Alter realized that many patients who received blood transfusions developed chronic hepatitis because of an unknown agent during the treatment procedure. Dr. Alter's research team noticed that infected patients can transmit the disease to chimpanzees. His further research revealed that this unknown infectious agent shows virus-like characteristics and thereby he defined a new form of chronic hepatitis which was introduced as "non-A, non-B" hepatitis. This discovery grounded the foundation of programs for screening blood donors which led to a global drop in transfusion-transmitted hepatitis.

Dr. Michael Houghton is currently the director of the Li Ka Shing Applied Virology Institute, Professor of Virology, and the Department of Medical Microbiology and Immunology in the Faculty of Medicine \& Dentistry at the University of Alberta, Canada. His discovery in 1989 established the new field of viral hepatitis research wherein blood safety, and the treatment of hepatitis $\mathrm{C}$ was aimed to be improved. Thanks to the developments in this area, today, viral infections can be treated in almost every affected patient. New screening tests were developed for blood donations as a result of their discovery. By 1992, the virus was virtually eliminated from the blood supply. This, in turn, led to a reduction in annually reported transmission by more than 80 percent by 1996 . Based on the discovery of the virus, antiviral therapies have also been developed that will cure 95 percent of hepatitis $\mathrm{C}$ carriers. This is the first chronic viral illness that can be cured.

\section{Nobel Prize in Chemistry}

The Royal Swedish Academy of Sciences awarded the 2020 Nobel Prize in Chemistry jointly to Jennifer A. Doudna and Emmanuelle Charpentier. This award was for their work on the development of a genome-editing tool (CRISPR/Cas9) which led to a breakthrough in biomedical research. This research provided a foundation for the later basic discoveries involving e.g., in plant research, for producing pest-, mold-, and drought-resistant crops. Furthermore, current cancer therapies are further advanced due to their discovery.

Dr. Emmanuelle Charpentier is the Scientific and Managing Director of the Max Planck Unit for the Science of Pathogens in Berlin, an independent institute that she founded together with the Max Planck Society. Her research focuses on fundamental mechanisms of regulation in processes of infection and immunity with a focus on Gram-positive bacterial human pathogens. Also, her work provided valuable insight into how RNAs and proteins control cellular processes at the transcriptional, post-transcriptional, and posttranslational levels. She also investigates interference systems 
in the defense against viruses and genetic elements (CRISPRCas9), small regulatory RNAs that interfere with pathogenic processes, protein quality control that regulates bacterial adaptation, physiology and virulence, basic principles of DNA replication and its effect on the bacterial, and vesicular interactions with the human host innate immunity.

Dr. Jennifer A. Doudna, who is the Li Ka Shing Chancellor's Chair in Biomedical and Health Sciences and a Howard Hughes Medical Institute investigator at the University of California, Berkeley, and the President and Chair of the Board of the Innovative Genomics Institute. Her research focuses on discovering and determining the mechanisms of a novel CRISPR-Cas9 system, which is guided by an RNA molecule to selectively bind a specific region in the DNA. The Cas9 protein which is capable to cut the DNA like a pair of molecular scissors, enabled precise alteration of the DNA. CRISPR-Cas9 allows scientists to rewrite DNA in human cells, with an extraordinary precision and efficiency. This groundbreaking power and versatility of these genetic scissors have opened new possibilities across medicine, biology, and agriculture. Several clinical trials that use CRISPR-based cell therapy to treat late-stage lung cancer and other diseases are underway.

\section{National Academy of Engineering}

The National Academy of Engineering (NAE) has announced the 2020 Ramos Founders award to Dr. Frances S. Ligler for the invention and development of portable optical biosensors, service to the nation and profession, and educating the next, more diverse generation of engineers. Dr. Ligler is the Lampe Distinguished Professor of Biomedical Engineering in the Joint Department of Biomedical Engineering in the College of Engineering at North Carolina State University and the School of Medicine at the University of North Carolina at Chapel Hill. Before then, she was at the US Naval Research Laboratory for 28 years, serving from 1995 as a Senior Scientist for Biosensors and Biomaterials. Currently working in the fields of biosensors, microfluidics, tissue-on-chip, and regenerative medicine, she has also performed research in biochemistry, immunology, and proteomics. The NAE is a private, independent, nonprofit institution that provides engineering leadership in service to the nation. Its members are among the world's most accomplished engineers. They provide the leadership and expertise for numerous projects focused on the relationships between engineering, technology, and the quality of life.

\section{National Academy of Medicine}

The National Academy of Medicine (NAM) has announced that Dr. Anthony S. Fauci, Director of National Institute of Allergy and Infectious Diseases (NIAID) will receive the
2020 Gustav O. Lienhard Award for Advancement of Health Care. Dr. Fauci is one of the nation's leading experts on infectious diseases which plays a scientifically grounded leadership role in our nation's response against the COVID-19 pandemic. He was one of the principal architects of the President's Emergency Plan for AIDS Relief (PEPFAR) and advised several US presidents. He was a pioneer in combating tuberculosis, HIV/AIDS, malaria, Ebola, and Zika across the country. Dr. Fauci has established a wide portfolio of basic and applied research on transplantation and immune-related illnesses At NIAID to prevent, diagnose, and treat infectious diseases. Dr. Fauci also received the NAM's first-ever Presidential Citation for Exemplary Leadership. Established in 1970 as the Institute of Medicine, NAM is an independent organization of eminent professionals from fields including health and medicine, as well as the natural, social, and behavioral sciences.

\section{Advancements in Academia}

A team of researchers led by Dr. Luiz E. Bertassoni, Associate Professor of Restorative Dentistry, School of Dentistry at Oregon Health \& Science University, recently introduced a 3D printing technique for biofabrication of miniaturized scaffolds with modular microcage architectures. The microcage designs were inspired by the interlocking toy building blocks. Ceramic-based composites of hydrogels with beta-tricalcium phosphate ( $\beta$-TCP) with feature sizes of $230-560 \mu \mathrm{m}$ thick walls were successfully fabricated through the stereolithographic layer by layer-based fabrication scheme. The designs allowed manufacturing scalability and ease of scaffold assembly and enabled facile patterning of thousands of geometric architectures for different hybrid therapeutic patterns. The microcages were incorporated with micro-granular hydrogels containing growth factors. The proposed assembly resulted in improved cell invasion into the core of the scaffolds to promote tissue formation as per the two times improvement in cell infiltration and three times larger vascularization in the composite scaffolds. These results suggest the great potential of the presented approach for bone and cartilage regeneration. This study was published in the journal Advanced Materials.

Dr. Achille Gravanis's research group in the Department of Pharmacology at School of Medicine of the University of Crete has recently published in npj Regenerative Medicine journal on the neural regeneration potential of a porous collagen scaffold for spinal cord injuries. Given the importance of the extracellular matrix in regulating neural stem cells (NSC), the group introduced a porous collagen environment for the NSC delivery and protection at the site of injury. The animal models of spinal cord injuries were suggestive of NSC protective effects and successful delivery of NSC at the site of injury by the proposed scaffolds. This observation was linked to the 
improvements in locomotion delivery according to a 12 -week study on the implanted animals which showed similar performance as the uninjured animals. Furthermore, The NSC-laden porous collagen grafts were characterized by neural regenerative capabilities in vivo and led to axonal elongation, and associated with lower levels of astrogliosis. The study proved the great promise offered by NSC encapsulated grafts for the treatment of the spinal cord lesions as an emerging therapeutic approach.

Another work by a research group directed by Dr. Rahmi Oklu, an Interventional Radiologist at Minimally Invasive Therapeutics Laboratory of Mayo Clinic, was published in the journal Advanced Materials on embolizing arteries for healing vasculatures. His research group aimed to reduce the risk of recanalization in the minimally invasive approaches for blocking the injured blood vessels. For this purpose, a nanocomposite hydrogel based on a decellularized cardiac extracellular matrix with bioactive and antimicrobial properties was engineered for their mechanical integrity, injectability, and retrievability. The embolization model based on the porcine iliac and renal arteries demonstrated that the proposed material platform provides a fibroinflammatory response with excellent vessel wall remodeling capability. Besides the superior biological function, $75 \%$ degradation in 2 weeks was reported that makes the material a potential candidate for clinical use as an embolic agent for treating vascular diseases.
In a study led by the research groups of Dr. Philip Wai-Yan Chiu and Dr. Liming Bian of the Chinese University of Hong Kong presented hyaluronic acid (HA)-catechol (Cat)-thiourea (NCSN)-based hydrogels for the treatment of gastric ulcers in rodent and porcine models. These hydrogels demonstrated ultrafast and $\mathrm{pH}$ independence gelation with a much lower oxidant concentration gelation and enhanced mechanical properties compared to classical bioadhesive hydrogels. A mixture of a precursor of HA-Cat and HA-NCSN hydrogel was applied topically onto the ulcer base using an endoscopic catheter, followed immediately by spraying the sodium periodate solution to induce the rapid in situ gelation. The HA-Cat-NCSN hydrogels promoted ulcer healing in vivo by enhanced cell proliferation and angiogenesis and upregulated genes associated with wound healing. These hydrogels may have restricted macromolecule transport and reduced the exposure of ulcer sites to catabolic enzymes in gastric juice, promoting the accumulation of cell-secreted pro-regenerative factors at ulcer sites that facilitated the ulcer healing process. This work was published in the journal Science Translation Medicine.

Publisher's Note Springer Nature remains neutral with regard to jurisdictional claims in published maps and institutional affiliations. 